d'une manière intempestive aux poussières de la gare laitière et lui fait perdre, par conséquent, tout le bénéfice de la pasteurisation initiale.

On doit admettre, et rien n'est plus indispensable, qu'une fois pasteurisé, le lait ne peut plus prendre contact avec l'atmosphère extérieure jusqu'au moment de la livraison au consommateur.

Dans le second, cas, on ne peut que déplorer un transvasement superflu, avec tous les risques de contamination et la perte de temps qui l'accompagnent, et bien plus encore la nécessité d'une nouvelle pasteurisation à la laiterie urbaine pour pallier à ces risques inévitables.

Ces quelques considérations nous permettront de conclure en disant que les plus belles réalisations techniques ou industrielles ne peuvent échapper au contrôle hygiénique et que, bien souvent, bon nombre de détails doivent lui être sacrifiés, dans l'intérêt de la santé publique.

\title{
CONTRIBUTION A L'ÉTUDE DU LAIT DE BUFFLESSE Production, propriétés et composition, dérivés \\ par
}

\author{
J. Y. PISSAREWSKY \\ Docteur ès Sciences
}

Lauréat de l'Académie d'Agrieulture de France

\section{(Suite) \\ 2。 LES QUALITÉS ET LA COMPOSITION DU LAIT DE BUFFLESSE}

\section{A. PROPRIÉtés PHYSIQUES}

Couleur. - Le lait de bufflesse est de couleur nettement blanche neigeuse : même après un repos prolongé. il ne présente jamais dans sa couche superficielle la teinte jaunâtre que l'on rencontre dans le lait de vache riche en graisse. Cela est dû au fait que la matière grasse du lait de bufflesse est plus riche en stéarine, ce qui lui prête une couleur plus claire que celle de la matière grasse du lait de vache. L'opacité du lait de bufflesse ne disparaît pas après l'addition d'une forte quantité d'eau, ce qui incite souvent les commerçants à la fraude, car ce lait présente difficilement la teinte bleuâtre, même après un fort mouillage.

Odeur et saveur. - On a parfois reproché au lait de bufflesse de présenter une odeur de musc, et nous en dirons la raison dans un instant. Affirmons tout de suite avec la plus grande énergie que, 
lorsque la mamelle est propre, le lait de bufflesse fraîchement trait ne possède aucune odeur sui generis qui puisse le différencier du lait de vache.

Cependant, il peut arriver, principalement en été, que le pis soit souillé de boue, lorsque les bufflesses (pour se protéger des insectes et de la chaleur) passent une grande partie de la journée vautrées dans ces flaques d'eau bourbeuses que les Italiens appellent "tonzi » et les Bulgares "kalista", et où elles déjettent leurs sueurs, leurs urines et leurs excréments. Alors il ne faut pas s'étonner, que le lait contracte une légère odeur musquée (qui est cependant spécialement appréciée des peuples orientaux), lorsque le vacher néglige de laver la mamelle avant la traite. Au contraire, nous avons maintes fois bu en Italie du lait de bufflesse récolté hygiéniquement, à l'étable ou au pâturage, dont nous ne pouvons faire que le plus grand éloge au point de vue de l'odeur et du goût, qui conviennent parfaitement à un palais occidental.

Nous trouvons même que le lait de bufflesse a une saveur beaucoup plus délicate, plus nuancée, moins fade que celui de la vache, notamment lorsqu'on l'emploie pour couper d'autres boissons comme le thé, le chocolat ou le café : "Le lait de bufflesse a un goût très apprécié, constate le $\mathrm{D}^{\mathrm{r}}$ VAIDA de Bucarest, et celui qui a bu du café au lait de bufflesse ne trouve plus aucune saveur au café au lait de vache. " La consommation directe du lait de bufflesse est très répandue en Proche-Orient, et c'est principalement ce lait que l'on boit au Caire, par exemple.

M. le Professeur Maccagnano donne la recette suivante, pour faire disparaître, dans le lait de bufflesse, le goût musqué, ou le goût médicamenteux dû à l'absorption accidentelle de certaines plantes avec la nourriture : «ajouter au lait un huitième d'eau bouillie et un peu de salpêtre, chauffer quelques minutes et refroidir brusquement en trempant le récipient dans une cuve d'eau froide. "

Densité. - La densité du lait de bufflesse varie entre les.limites extrêmes suivantes :

$\mathrm{D}$ maxima $=1.037$ trouvée par $\mathrm{G}$. FASCETTI.

$D$ minima $=1.026,5$ rencontrée par BAINTNER et IRK dans un lait provenant d'une bufflesse en fin de lactation (Hongrie).

Le $\mathrm{D}^{\mathrm{r}} \mathrm{B}$. MAYMONe a trouvé, pour 42 échantillons de lait de bufflesse livrés à la fromagerie sociale de Battipaglia et provenant de différents élevages de la région (balance de Mohr-Westphal, chiffres ramenés à $15^{\circ} \mathrm{C}$.), un poids spécifique moyen de 1,0331 , soit une $\mathrm{D}$ moyenne $=1033,1$. (Italie.)

Les nombreuses prises de densité (lactodensimètre) auxquelles nous nous sommes livré, tant dans la région de Battipaglia, que dans d'autres localités de la plaine de Salerne, ainsi que près de Naples et 
à la ferme de Tor' Mancina de l'Institut Expérimental Zootechnique de Rome, nous ont donné, pour 27 expériences, une $\mathrm{D}$ moyenne $=$ 1032,8, chiffre comparable à ceux trouvés par RIEveL $(1033,9)$, Stazi (1033), Pizzi (1032), Groliani (1032). Pour toutes ces données on a tenu compte de la correction à $15^{\circ}$.

Rappelons que la densité du lait de vache est analogue, et varie (cf. Dornic et Chollest) de 1026 à 1036. Nous devons attribuer cette analogie au fait que le lait de bufflesse contient en plus forte proportion relative (of. Propriétés chimiques) à la fois des substances de densité basse (matière grasse) et des substances de haute densité (matière prot. surtout) : finalement la densité du lait ne change guère.

Tension superficielle absolue. - Constante physique définitivement exprimée en $\mathrm{mgr} . / \mathrm{mm}^{2}$.

Lait de vache $($ Ko BLER $) \ldots \ldots \ldots \ldots \ldots \ldots \ldots \quad 5,060$ à 5,726

Lait de bufflesse (MAXMONE) $\ldots \ldots \ldots \ldots \ldots \ldots 4,419$ à 5,509

Viscosité relative. - Le lait de bufflesse a une très grande viscosité et "colle" aux récipients, aux lactodensimètres, etc.

$\begin{array}{lll}\text { Lait de vache (Cavazzoni) } \ldots \ldots \ldots \ldots \ldots \ldots \ldots \ldots & 1,67 \text { à } 2,03 \\ \text { Lait de chèvre (Cavazzoni) } & \ldots \ldots \ldots \ldots \ldots \ldots \ldots & 2,01 \text { à } 2,15 \\ \text { Lait de bufflesse (Maymone) } & \ldots \ldots \ldots \ldots \ldots \ldots \ldots & 2,33 \text { à } 2,97\end{array}$

(Viscosité de l'eau distillée $=1$ à $15^{\circ} \mathrm{C}$.)

Constante cryoscopique Raoult et acidité normale. - Un lait aura une température de congélation d'autant plus basse, que son degré d'acidité sera plus fort. D'autre part le mouillage augmente d'autant plus la constante cryoscopique en rapprochant $\Delta$ de $0^{\circ}$ que la quantité d'eau ajoutée est plus forte. (Détection des fraudes.)

Lait de vache

$$
\begin{aligned}
& \Delta=-0^{\circ} 55 \text { à }-0^{\circ} 56 \\
& \Delta=-0^{\circ} 56 \text { à }-0^{\circ} 57 \\
& \Delta=-0^{\circ} 56 \text { à }-0^{\circ} 59 \\
& \Delta=-0^{\circ} 57 \text { à }-0^{\circ} 60
\end{aligned}
$$

Lait de chèvre

Lait de bufflesse

Le Professeur Maymone a étudié 15 échantillons de lait, d'acidité $8^{\circ} 1$ à $9^{\circ} 9$ Soxhlet-Henkel à peine supérieure à celle du lait normal 70 S.-H. "Dans un lait de bufflesse analysé au Laboratoire de la Chaire ambulante d'agriculture de Salerne, avec $18^{\circ} 6$ d'acidité Soxhlet-Henkel, le point de congélation s'est abaissé à $0^{\circ} 71$ et, dans ce cas, l'excessif abaissement de l'indice cryoscopique est dû évidemment à la plus grande concentration moléculaire déterminée par la formation de l'acide lactique à partir du lactose. ”

Notons que la graduation d'acidité Dornic (soude N/9 et phénolphtaléine comme réactif coloré) est la plus employée en France puis- 
qu'elle correspond exactement à 10 fois le nombre de grammes d'acide lactique par litre :

Lait de chèvre $\quad . \quad 13^{\circ}$ à $16^{\circ}$ Dornic ( 1 gr. 3 à 1 gr. 6 d'acide lactíque par litre) Lait de vache.... $17^{\circ}$ à $21^{\circ}$ Dornic ( 1 gr. 7 à 2 gr. 1 d'acide lactique par litre) Lait de bufflesse . $\quad 18^{\circ}$ à $22^{\circ}$ Dornic ( 1 gr. 8 à 2 gr. 2 d'acide lactique par litre) selon les dosages que nous avons effectués sur 8 échantillons de lait de mélange provenant de divers élevages des environs de Battipaglia (Laboratoire de la Federazione Fascista degli Agricoltori, 30 juillet 1934).

Constante réfractométrique. - Le réfractomètre permet de déceler pour le lait de chaque espèce animale une constante spécifique, ce qui est un excellent moyen pour la détermination du mouillage. L'indice réfractométrique est de :

Vache

Chèvre

Brebis
1,3430 à 1,3445

1,3433 à 1,3457

1,3455 aे 1,3465

(D'après A. Monvoisin : lactosérum acétique Villiers et Bertault.) Bufflesse 1,3412 à 1,3465

(D'après MAYMONE : lactosérum chloruro-calcique Ackermann.)

Au moment de publier ce travail, nous sommes avisé que le Laboratoire agricole de Caserte vient d'entreprendre, pour le lait de bufflesse, des recherches réfractométriques comparatives par la méthode chloruro-mercurique d'Ambühl et Weiss et par celle silicotungstique récemment préconisée par notre distinguée collaboratrice $\mathrm{M}^{\text {me }} \mathrm{D}^{\mathrm{r}} \mathrm{R}$. WEISSMANN-KaRLin (Etude de la réfractométrie du lait de vache, thèse Faculté Sc. Nancy, 1933). Les résultats de ces recherches feront l'objet d'une étude ultérieure:

Conductibilité électrique. - Le $\mathrm{D}^{\mathrm{r}}$ BINAgLi indique les chiffres suivants, établis sur 36 échantillons de lait pur (Pont Wheatstone, méthode Kohlrauch) : brebis.

$\mathrm{K}=49,43 \times 10^{-4}$ à $51,72 \times 10^{-4}\left(\mathrm{ohms}^{-1}\right)-$ pour le lait de

$\mathrm{K}=47,04 \times 10^{-4}$ à $49,96 \times 10^{-4}\left(\mathrm{ohms}^{-1}\right)-$ pour le lait de chèvre.

$\mathrm{K}=47,97 \times 10^{-4}$ à $49,98 \times 10^{-4}\left(\mathrm{ohms}^{-1}\right)-$ pour le lait de vache.

L9 Professeur Maymone, qui a étudié cinq laits individuels de bufflesse et un lait collectif (mélange des traites de quatre bufflesses), a trouvé des chiffres variant entre :

$\mathrm{K}=32,66 \times 10^{-4}$ (bufflesse au $319^{\mathrm{e}}$ jour de sa quatrième lactation) et - 
$\mathrm{K}=50,26 \times 10^{-4}$ (bufflesse au $13^{\mathrm{e}}$ jour de sa troisième lactation - à la température de $20^{\circ} 5 \mathrm{C}$.

Cette dernière valeur doit être attribuée probablement à la richesse éventuelle en sels minéraux d'un lait au $13^{e}$ jour de lactation, qui gardait encore certaines des propriétés du colostrum.

\section{B.:PROPRIÉTÉS CHIMIQUES}

Matière grasse. - "La richesse en matière grasse est la caractéristique principale du lait de bufflesse. Il est normalement plus riche en graisse que les laits de vache, de chèvre, ou de brebis.

"La teneur en graisse du lait de bufflesse, comme celle des autres laits, varie beaucoup sous l'influence de l'individualité, de l'âge, de la période de lactation, de l'alimentation, de la vie sexuelle, de la saison, ete.

"Le fort contenu en graisse rencontré dans les laits analysés au printemps et en été doit être surtout attribué au fait, que la plupart des bufflesses, en ces saisons, sont en période de fin de lactation.

"Chez une même bufflesse peuvent exister aussi des variations

TABLEAU II.

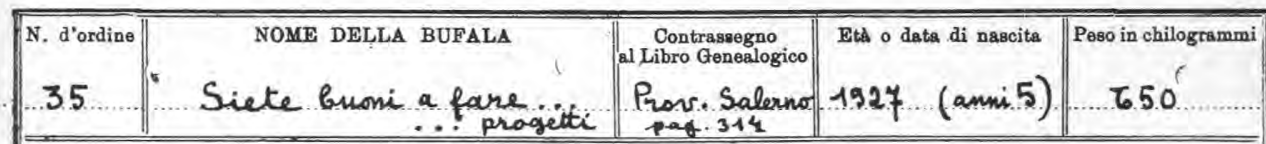

Data del parto 2. Genmaio 19.32. Data dell'ultima mungitura ..13 Ott. 32. (Sesso del nato: maschio, Matricola of 1 ) RISULTATI DEL ${ }^{32}$ CONTROLLO

\begin{tabular}{|c|c|c|c|c|c|c|}
\hline \multirow{2}{*}{ 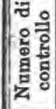 } & \multicolumn{2}{|c|}{ DATA } & \multicolumn{3}{|c|}{ Chilogrammi di latte } & \multirow{2}{*}{$\begin{array}{c}010 \\
\text { di } \\
\text { grasso }\end{array}$} \\
\hline & Mese & Gior. & Sera & Mattina & TOTALE & \\
\hline 1 & Genn & 15 & 180 & 2300 & & 5, \\
\hline 2 & 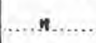 & 30. & 26 & ry & & 5,8 \\
\hline 3 & Felar. & 15 & 2 & 9 lod & & 6,2 \\
\hline 4 & Marzo & 6 & 3. & 2600 & 5900 & 6,3 \\
\hline 5 & $\ldots *$ & 16 & & 2. 700 & $\infty$ & 6,5 \\
\hline 6 & Aprile & 2 & & 3.400 & $\infty$ & 6,5 \\
\hline 7 & 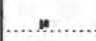 & 16 & & 3 thes & 6400 & 6,8 \\
\hline 8 & Maggi & 6 & & .9400 & & 7.2 \\
\hline 9 & $\therefore$ & 1.5 & & 3.300 & & $.6,9$ \\
\hline
\end{tabular}

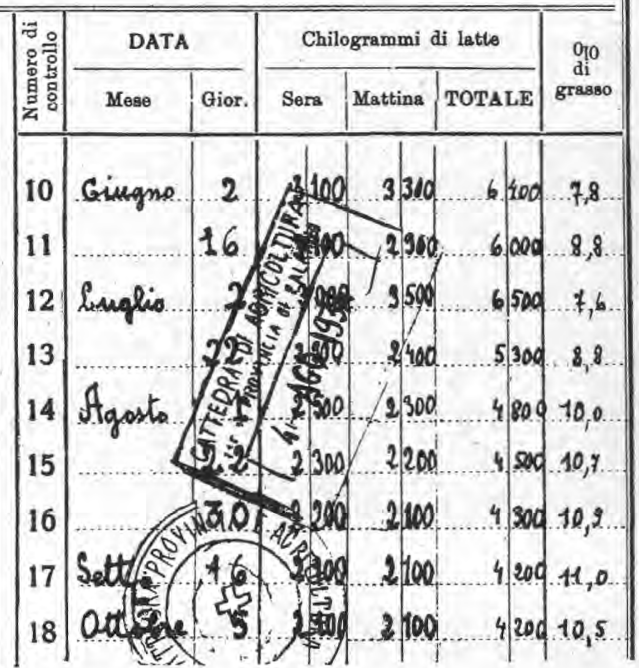


sensibles dans la teneur en matière grasse du lait, d'un jour à l'autre, sans l'intervention de causes modificatrices apparentes. "

C'est ainsi que le Professeur Maymone rencontre dans le lait d'une bufflesse en deuxième lactation, ayant mis bas depuis 15 jours (octobre 1922), un minimum de 5,95\% de matière grasse ; et chez une autre bufflesse en fin de lactation, ayant mis bas depuis plus d'un an, un maximum de $12,13 \%$ de matière grasse.

La race a également une influence indéniable : à nombre de têtes égal et toutes conditions du régime d'élevage étant égales d'ailleurs, les expériences poursuivies par W. Sмптн à la Military Dairy Farm of Kirkee (Indes britanniques) ont décelé chez la race Gujerati une teneur moyenne de matière grasse (pour toute la période de lactation) de $8,4 \%$, et chez la race de Delhi de $7,5 \%$.

Il y a aussi des variations, au courant de la même journée : la traite du matin est généralement moins riche que celle du soir. "Dans l'Inde, Megrit et Mann Harold ayant suivi de janvier 1908 à janvier 1909 la production laitière des 24 bufflesses de la Vacherie municipale de Poona, ont trouvé un contenu moyen en graisse, pendant toute la période considérée, de $7,60 \%$ dans le lait trait le matin et de $7,92 \%$ dans le lait trait le soir. "

Un maximum remarquable a été rencontré pour la matière grasse par Levine, chez une bufflesse chinoise du Christian College de Canton, dont le lait avait le taux, pour le moins extraordinaire, de $17 \%$, soit 170 grammes par litre! (dernier mois de lactation).

Outre les trois laits qui ont fait l'objet de notre part d'une analyse complète à l'Ecole de Laiterie de Nancy, nous avons effectué, dans divers laboratoires officiels ou privés, en Italie (juillet-août 1934) un total de 35 déterminations de la matière grasse par les méthodes Gerber ou Lindström (Colibri) ou encore par celle, plus précise, de l'extraction par l'éther. Nous avons réuni ainsi 38 chiffres dont la valeur varie de:

$$
6,47 \% \text { à } 12,3 \%
$$

et qui se rapportent tantôt à des laits individuels, tantôt à des laits de mélange. Nous avons remarqué que, comme pour les vaches, les chiffres les plus élevés correspondent toujours, dans les laits de mélange, à une alimentation améliorée (bons pâturages ou stabulation). Le tableau ci-après comprend huit chiffres de matière grasse déterminés pour des laits de mélange provenant de différents élevages semi-extensifs de la plaine du Sele (30 juillet 1934). 
TABLEAU III.

Confederazione Provinciale Fascista degli Agricoltori Consorzio Produttori Latte Della Piana del Sele Battipaglia

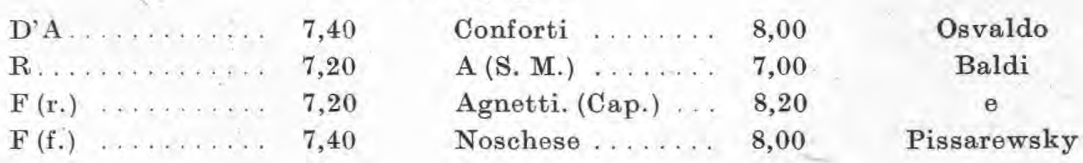

Le maximum $12,3 \%$ a été trouvé par nous dans le lait $\mathrm{n}^{\circ} 1$, analysé à Nancy, qui provenait d'une bufflesse en pleine force de l'âge ( 8 ans), en période avancée de lactation ( $8^{\mathrm{e}}$ mois), alimentée à l'étable d'une façon rationnelle (régime d'hiver).

Le Dr G. Colangelo, lors de la visite que nous lui avons faite à la Chaire ambulante d'Agriculture de Salerne, a bien voulu nous confier un extrait-type du Registre de contrôle laitier et beurrier des bufflesses (reproduit au tableau II) grâce auquel nous avons tracé ce graphique IV des variations du taux de matière grasse, chez une lai-

TABLEAU IV.

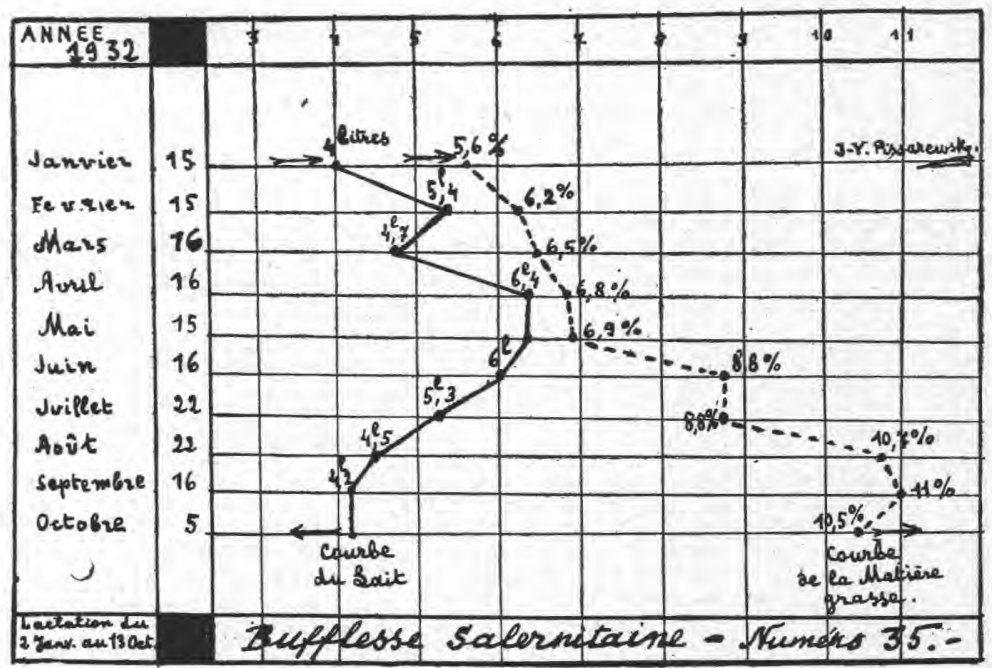

tière moyenne ( ( Siete buoni a fare... progetti $) \mathrm{n}^{\circ} 35$ ), en fonction du rendement en lait quotidien, de la saison de l'année, et de l'éloignement dans le temps à partir du part. Ici, très nettement, le lait est pauvre en graisse au commencement de la lactation et très riche à la fin.

Par sa richesse plus grande en stéarine, la matière grasse du lait de bufflesse est moins dense que celle du lait de vache : M. le Professeur MAYmone en a étudié les propriétés physico-chimiques, et 
nous reproduisons ci-dessous les résultats auxquels ont abouti ses expériences :

\begin{tabular}{|c|c|}
\hline Poids spécifique à $100^{\circ} \mathrm{C} . \ldots$ & 0,8754 \\
\hline Température de fusion $\ldots \ldots \ldots \ldots \ldots$ & $32^{\circ} 0 \mathrm{C}$. à $39^{\circ} 0 \mathrm{C}$. \\
\hline Température de solidification $\quad \ldots \ldots \ldots \ldots$ & $24^{\circ} 0 \mathrm{C}$. à $28^{\circ} 0 \mathrm{C}$. \\
\hline Température de fusion des acides gras ..... & $42^{\circ} 0$ C. à $44^{\circ} 0$ C. \\
\hline Temp. de solidification des acides gras .... & $39^{\circ} 5 \mathrm{C}$ \\
\hline Indice réfractométrique à $30^{\circ} \mathrm{C} . \quad \ldots \ldots \ldots$ & 45,00 à 46,00 \\
\hline Indice de saponification $\ldots \ldots \ldots \ldots \ldots$ & 222,00 aे 225,00 \\
\hline Indice Reichert-Meiss1 $\ldots \ldots \ldots \ldots \ldots \ldots$ & 31,00 à 33,00 \\
\hline Indice Hehner $\ldots \ldots \ldots \ldots$ & 86,10 à 87,17 \\
\hline Indice Polenske $\ldots \ldots \ldots \ldots \ldots \ldots \ldots \ldots$ & 1,80 à 2,00 \\
\hline Proportion relative d'acétyle . . . . . . . . . . & $14,0 \%$ à $15,0 \%$ \\
\hline Proportion relative d'iode $\ldots \ldots \ldots \ldots \ldots$ & $36,0 \%$ à $38,0 \%$ \\
\hline Substances non saponifiables . . . . . . . . . & $1,2 \%$ à $1,5 \%$ \\
\hline
\end{tabular}

Extrait sec et extrait dégraissé. - La grande richesse du lait de bufflesse en extrait sec permet de faire, avec le même volume de lait complet, une quantité de fromage gras presque double de celle obtenue avec du lait de vache. Si bien que les fromageries, en Italie, par exemple, paient souvent deux fois plus cher l'hectolitre de lait de bufflesse que l'hectolitre de lait de vache! (cf. sous-chapitre suivant).

Nous avons déterminé l'extrait sec $\mathrm{E}$. $\mathrm{s}$. des échantillons de lait dont nous disposions, en évaporant dans une étuve, à $65^{\circ} \mathrm{C}$., jusqu'à l'obtention d'un poids constant, $10 \mathrm{~cm}^{3}$ de lait, mis dans une capsule tarée, et coagulé, selon la méthode de Hinard, par 3 gouttes de solution formolée-acétique.

Nos résultats sont absolument comparables avec ceux obtenus par différents auteurs (après déduction préalable du bichromate de potassium dans les laits $n^{0} 1$ et $n^{0} 3$ ).

Diaconu (Roumanie) : 20,45\% (moyenne pour le lait de 3 bufflesses analysé à intervalles réguliers durant une lactation entière).

BaINTER et IRK (Hongrie) : 17,90\% (moyenne de 200 analyses). BaINTER et IRK (Hongrie) : 27,05\% (bufflesse en fin de lactation). Maymone (Italie) : 18,39\% (moyenne de 13 déterminations).

Marsella (Italie) : $19 \%$.

PissarewSky (Italie): $23,84 \%$ (lait $n^{0} 1$ individuel, $8^{\mathrm{e}}$ mois de lactation).

Pissarewsky (Italie) : $22,80 \%$ (lait $\mathrm{n}^{\circ} 2$ collectif de 25 bufflesses).

Pissarewsky (Italie) : $22,84 \%$ (lait $\mathrm{n}^{\circ} 3$ collectif de 25 bufflesses (hiver)).

PaPpel et Hogan (Egypte) : 17,91\% (nombreuses analyses au Caire). 
Megatt et Mann' Harold (Inde) : $18,30 \%$ (moyenne en saison froide).

L'extrait dégraissé Ed. = Es - M. gr. est pratiquement la somme de lactose hydraté + cendres réelles + matières protéiques totales : on ne compte généralement pas les acides citrique et indosé.

C'est ce qu'on appelle la constante L. Gros qui n'a d'ailleurs guère de valeur pour déterminer le mouillage, même dans le lait de vache, auquel l'affecte son auteur : puisque, ainsi que l'a notamment démontré M. J. CERF (thèse Fac. Sciences, Nancy, 1932), les variations peuvent aller de 80 à 110 grammes par litre (quoique plus habituellement comprises entre 85 et 90 grammes). D'après les chiffres qui sont en notre possession : - l'extrait dégraissé se maintient habituellement chez la bufflesse entre 95 et 110 grammes, mais peut varier de 90 à 120 grammes par litre de lait.

L'extrait dégraissé et l'extrait sec se calculent, comme on sait, empiriquement (pour le lait de vache) par la formule bien connue de Fleischmann et celle, tout aussi excellente de notre maître $M$. BouIn et de M. Gobert, que nous citons à titre de documentation (donne l'extrait pour $100 \mathrm{~cm}^{3}$ de lait) :

$$
\mathrm{Ed}=0,2 \mathrm{~b}+\frac{0,8 \mathrm{D}}{3} \quad \mathrm{Es}=1,2 \mathrm{~b}+\frac{0,8 \mathrm{D}}{3}
$$

Cependant ni l'une ni l'autre de ces formules, pas plus d'ailleurs que celle proposée par 0 . LAXA pour le lait de brebis, ne peuvent être appliquées au lait de bufflesse, pour lequel elles donnent des valeurs généralement beaucoup trop élevées en pourcentage. Des résultats assez approximatifs s'obtiendraient, au contraire, avec les formules proposées par RICHMOND (in B. MAYMONE) et par PAPPEL :

$$
\mathrm{Ed}=\frac{\mathrm{b}}{5}+\frac{\mathrm{D}}{4} \quad \mathrm{Es}=\mathrm{b}+\frac{\mathrm{b}}{5}+\frac{\mathrm{D}}{4}
$$

La formule de PAPPEL donne des résultats approximatifs par défaut qui ne nous ont pas paru encore suffisamment précis : e'est pourquoi nous avons l'honneur de proposer de lui substituer la formule suivante, plus exacte :

$$
\mathrm{Ed}=\frac{\mathrm{b}+\mathrm{D}}{4,12} \quad \mathrm{Es}=\mathrm{b}+\frac{\mathrm{b}+\mathrm{D}}{4,12}
$$

Dans toutes les formules ci-dessus " $\mathrm{b}$ 》 est le taux de la matière grasse pour $100 \mathrm{~cm}^{3}$ de lait et ( $\mathrm{D}$ 》 le degré densimétrique à $15^{\circ} \mathrm{C}$. (par exemple si la densité est $=1033,1$, on a $\mathrm{D}=33,1$ ).

Pour pouvoir juger de la valeur définitive de la formule que nous préconisons, il faudrait la vérifier pour une centaine d'analyses, par exemple. Or, nous n'avons pu effectuer les calculs que pour les 


\begin{tabular}{|c|c|c|c|c|c|c|c|c|}
\hline \multirow[t]{2}{*}{$\begin{array}{c}\text { Expérimen - } \\
\text { tateurs }\end{array}$} & \multicolumn{3}{|c|}{ Analyse directe } & \multicolumn{2}{|c|}{$\begin{array}{c}\text { Formule } \\
\text { Pappel }\end{array}$} & \multicolumn{2}{|c|}{$\begin{array}{c}\text { Formule } \\
\text { Pissarewsky }\end{array}$} & $\begin{array}{c}\text { Formule } \\
\text { Bouin et Go- } \\
\text { bert (b́tablie } \\
\text { pour le lait } \\
\text { de vache) }\end{array}$ \\
\hline & $\mathrm{D}$ & G & $\mathrm{Ed}$ & Ed & Ecarts & Ed & Ecarts & Ecarts \\
\hline Giuliani .... & 31,3 & 9,02 & 9,31 & 9,63 & $0,32+$ & 9,78 & $0,47+$ & $10,140,83+$ \\
\hline Pizzr $\ldots . \ldots \ldots$ & 32,0 & 7,95 & 9,85 & 9,59 & $0,26-$ & 9,69 & $0,16-$ & $10,120,27+$ \\
\hline RIEVel $\ldots \ldots$. & 33,9 & 7,40 & 9,99 & 9,95 & $0,04-$ & 9,92 & $0,07-$ & $10,520,53+$ \\
\hline Stazi $\ldots .$. & 33,0 & 8,27 & 10,27 & 9,90 & $0,37-$ & 10,01 & $0,26-$ & $10,450,18+$ \\
\hline Pissarew ky & 35,0 & 12,30 & 11,54 & 11,21 & $0,33-$ & 11,48 & $0,06-$ & $11,790,25+$ \\
\hline Pissarewsky & 35,0 & 11,50 & 11,30 & 11,05 & $0,25-$ & 11,28 & $0,02 .-$ & $11,630,33+$ \\
\hline PISSAREWSKY & 35,2 & 11,50 & 11,34 & 11,10 & $0,24-$ & 11,33 & $0,01-$ & $11,680,34+$ \\
\hline
\end{tabular}

sept analyses dont nous possédions tous les éléments nécessaires ; d'autre part, en consultant le tableau ci-contre, il paraît nécessaire d'éliminer a priori l'analyse de GIULIANI qui doit être erronée puisqu'elle donne des résultats excessifs et aberrants tant par la formule de MM. BouIn et GoBert, que par celle de PAPPEL et par la nôtre.

Ceci dit, les résultats comparatifs pour les analyses de STAzI, Pizzi, Rievel, et nos trois analyses personnelles s'établissent comme suit :

Par la formule Bouin et Gobert :

Tous les écarts sont positifs et s'échelonnent entre $+0,18$ et $+0,53$, pour $100 \mathrm{~cm}^{3}$ de lait.

Sommэ algébriquэ des six écarts . . . . . . . . $=+1,900$

Moyenne d'écart .................... $=+0,316$

Correction du poids d'Ed par litre : déduire $3 \mathrm{gr} .1 \ell$.

Par la formule Pappel :

Tous les écarts sont négatifs et s'échelonnent entre - 0,04 et $-0,37$, pour $100 \mathrm{~cm}^{3}$ de lait.

Somme algébrique des six écarts $\ldots \ldots \ldots \ldots \ldots \ldots=-1,490$

Moyenne d'écart $\ldots \ldots \ldots \ldots \ldots \ldots \ldots \ldots \ldots \ldots=-0,248$

Correction du poids d'Ed par litre : ajouter 2 gr. 48.

Par la formule Pissarewsky :

Tous les écarts sont négatifs et s'échelonnent entre $-0,01$ et $-0,26$, pour $100 \mathrm{~cm}^{3}$ de lait.

Somme algébrique des six écarts $\ldots \ldots \ldots \ldots \ldots \ldots=-0,580$

Moyenne ..................... $=-0,0,096$

Correction du poids d'E.d par litre : il suffira d'ajouter 0 gr. 96. 
Substances azotées. - Comme dans tous les laits, il existe dans le lait de bufflesse de la caséine, de la lactalbumine, de la lactoglobuline et, en outre, divers composés non protéiques, tels que des acides aminés, des urates, etc.

Nous avons déterminé l'Azote total, pour les échantillons que nous avons analysés à Nancy, par la méthode ordinaire Kjeldahl; nous avons calculé empiriquement la matière protéique totale en multipliant l'azote par 6,35 ; et nous avons obtenu la caséine par le même procédé en multipliant la matière protéique totale par 0,08 (1). Ce qui a donné :

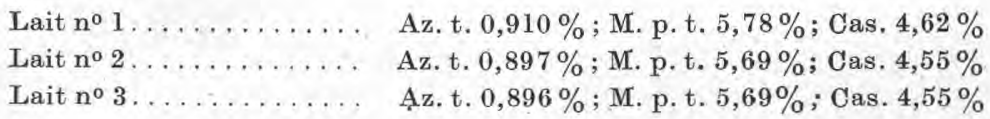

Diaconu (Roumanie) a trouvé $7,33 \%$ de substance azotée totale, en moyenne. Il a déterminé ce chiffre en déduisant de l'extrait sec total la somme de tous les autres composés trouvés par des analyses successives.

Batntner et Irk (Hongrie) ont trouvé une variation de $4,013 \%$ à $6,840 \%$ du début à la fin de la lactation chez une bufflesse donnée (matière protéique totale).

Levine, à Canton, a trouvé $6,05 \%$ de matière protéique totale dans un lait très riche en graisse et en résidu sec.

Gruliani (Italie) donne une moyenne, exprimée en caséine, de $3,99 \%$.

Rimini assigne au lait de bufflesse $3,62 \%$ de caséine et $0,73 \%$ d'albumine.

Stazi assigne au lait de bufflesse $3,63 \%$ de caséine et $0,73 \%$ d'alb.

Marsella assigne au lait de bufflesse $4,30 \%$ de caséine et $1,30 \%$ d'albumine.

Martinoli assigne au lait de bufflesse $3,10 \%$ de caséine et $0,06 \%$ d'albumine.

Fleischmann assigne au lait de bufflesse 4,29\% de caséine et $0,61 \%$ d'albumine.

Gordp-Besanez assigne au lait de bufflesse $4,21 \%$ de caséine et $1,30 \%$ d'albumine.

Le Professeur B. Maymone a procédé à des recherches par la méthode classique Orla Jensen qui ont donné, pour 13 échantillons différents de lait provenant de bufflesses de la plaine de Salerne, les résultats suivants : Le contenu maximum de caséine a été de $3,38 \%$, contre un minimum de $2,77 \%$ et une moyenne de $3,04 \%$. Le contenu moyen en lactalbumine + lactoglobuline a été de $0,90 \%$, avec un maximum de $1,22 \%$ et un minimum de $0,67 \%$. Le contenu moyen

(1) Ce n'est qu'approximatif, par défaut, dans les laits des bufflesses fraîchement vêlées, etc. 
en substances azotées non protéiques a été de $0,24 \%$, avec un maximum de $0,32 \%$ et un minimum de $0,19 \%$.

On voit, par conséquent, de ce qui précède, que la caséine (qui nous intéresse tout particulièrement puisque c'est surtout elle qui est coagulée par la présure lors de la fabrication du fromage) varie, chez la bufflesse, entre les limites approximatives suivantes :

de 3,00 à 5,$00 ;$ moyenne $4,00 \%$ - contre :

de 2,50 à 4,00 ; moyenne $3,00 \%$ chez la vache,

quand on considère des laits purs et normaux. Le lait de bufflesse est done plus fromager que celui de vache, équivalent ou légèrement supérieur à celui de chèvre, mais moins fromager cependant que celui de brebis (moyenne, caséine $=5 \%$ ).

Nous parlerons de la fabrication des fromages de bufflesse dans le sous-chapitre suivant.

Constante Cornalba. - Le Dr G. CoRnalBA, après avoir observé que les matières solubles du lait ne variaient que de 5,9 à $6,6 \%$ chez la vache, en avait déduit qu'elles constituaient l'élément le plus constant ainsi que le plus propre à l'évaluation du mouillage. La constante Cornalba est l'expression extrait sec moins (matière grasse, plus caséine), autrement dit :

\section{E.d. - Caséine.}

Nous citons ci-dessous le calcul de cette constante d'après les chiffres concernant le lait de bufflesse de trois auteurs précités : Rimini, Stazi, Marsella (qui sont les seuls analystes ayant déterminé à la fois la caséine pure et le résidu sec dégraissé) èt d'après les chiffres résultant de nos expériences personnelles.

\begin{tabular}{|c|c|c|c|}
\hline & Extr, $d$ & - Caséine & $\begin{array}{c}=\text { C.te } \\
\text { Cornalba }\end{array}$ \\
\hline RIMINI & 10,37 & $-3,62$ & $=6,75$ \\
\hline Stazi ... & 10,27 & $-3,63$ & $=6,64$ \\
\hline MARSELLA & 10,90 & $-4,30$ & $=6,60$ \\
\hline Lait $n^{0} 1 \ldots$ & 11,54 & $-4,62$ & $=6,92$ \\
\hline Lait $n^{\circ} 2$ & 11,34 & $-4,55$ & $=6,79$ \\
\hline Lait $n^{\circ} 3$. & 11,34 & $-4,55$ & $=6,75$ \\
\hline
\end{tabular}

D'après les chiffres ci-dessus, il y aurait une variation entre 6,6 et 6,92 ; il est à remarquer, en outre, que, chez une bufflesse donnée, le Cornalba diminue très probablement en cours de lactation, parallèlement au lactose.

Lactose. - Pour la détermination du lactose dans le lait de bufflesse, tous les auteurs que nous avons consultés (du moins ceux qui indiquent les méthođes qu'ils ont suivies) ont utilisé la propriété que possède ce sucre de réduire la liqueur cupro-potassique 
de Fehling; ou encore, ils ont fait cette détermination gravimétriquement.

Nous croyons donc à bon escient être le premier à avoir utilisé la méthode polarimétrique, qui, grâce aux perfectionnements remarquables qu'elle a reçus ces dernières années, est désormais, d'après l'expérimentation faite pour le lait de vache, aussi exacte que les méthodes chimiques les plus sûres, ainsi que l'affirment à juste raison Georges BruHat, auteur du "Traité de polarimétrie " (1930) et G. ADLE (thèse Doct. Sciences, Nancy, 1931).

Cependant, nous devons faire cette réserve que nous ignorons si le lait de bufflesse ne renferme pas aussi, comme certains autres laits (femme, ânesse, jument), des substances à pouvoir rotatoire, autres que le lactose, ayant pu modifier la déviation du rayon lumineux et fausser nos lectures au polarimètre. Nous n'avons pu contrôler cette hypothèse, dont la vérification eût demandé des recherches complémentaires incompatibles avec la faible quantité de lait dont nous disposions.

Nous nous sommes servi, pour la défécation du lait par précipitation des albuminoïdes et lipoïdes, successivement du réactif picro-acétique d'Esbach et du réactif silico-tungstique (milieu acétique) de M. le Prof. Bourn. C'est avec ce dernier réactif que nous avons obtenu, pour le lait de bufflesse, les sérums les plus limpides, en employant cependant une dose de ce réactif double de celle ordinairement employée pour le lait de vache. En effet, nous savons que les substances protéique et grasse sont en quantité relativement plus forte dans le lait de bufflesse : aussi faut-il, pour précipiter tout le coagulum, employer une plus grande dose dè réactif. Si le lait est bichromaté, on ajoute quelques gouttes d'acétate neutre de plomb, afin de précipiter le bichromate de potasse sous forme de chromate de plomb. Par ce procédé nous avons eu des liquides parfaitement transparents après une seule filtration, et très favorables à l'examen polarimétrique.

Nous croyons utile de rappeler ici le mode de préparation et d'emploi du réactif silico-tungstique, d'après la technique que préconise M. BouIn pour le lait de vache, et de présenter les modifications que nous avons introduites pour le lait de bufflesse :

10 grammes d'acide silico-tungstique (préparé par exemple par méthode Pichon-Vendeuil) sont dissous dans un peu d'eau acidulée par l'acide acétique ; on ajoute $25 \mathrm{~cm}^{3}$ d'acide acétique cristallisable et on complète avec de l'eau distillée : quantité suffisante pour $500 \mathrm{~cm}^{3}$. 
Vache

M. Bouin opèré

somme suit :

Lait de vache ..........

Réactif Bouin ...........

Eau distillée qu.s. p.

Bufflesse

Nous avons opéré

comme suit:

$25 \mathrm{~cm}^{3} \quad$ Lait de bufflesse ....... $25 \mathrm{~cm}^{3}$

$10 \mathrm{~cm}^{3} \quad$ Réactif Bouin ........ $20 \mathrm{~cm}^{3}$

$50 \mathrm{~cm}^{3} \quad$ Eau distillée qu.s. p. .... $100 \mathrm{~cm}^{3}$

Soit V le volume du coagulum pour $100 \mathrm{~cm}^{3}$ de lait de bufflesse primitif (dont on n'a employé que $25 \mathrm{~cm}^{3}$, soit $1 / 4$ ) :

$\mathrm{V}=\frac{\text { matière grasse p. } 100 \mathrm{~cm}^{3}}{0,93}+\frac{\text { matière protéique totale } \mathrm{p} \cdot 100 \mathrm{~cm}^{3}}{1,35}$

0,93 étant la densité admise de la matière grasse à $15^{\circ}$ C. ; 1,35 étant la densité admise de la matière protéique totale à $15^{\circ} \mathrm{C}$.

Après avoir ajouté le réactif et l'eau de dilution, les $100 \mathrm{~cm}^{3}$ de mélange obtenus donnent, par précipitation du coagulum, et après filtration préalable, un volume $U$ de solution déféquée polarisable :

$$
\mathrm{U}=100 \mathrm{~cm}^{3}-\frac{\mathrm{V}}{4}
$$

Le poids $\mathrm{P}$ de lactose, dans les $\mathrm{U}$ centimètres cubes de filtrat (calculés comme ci-dessus), serait, pour une déviation polarimétrique enregistrée $A$, obtenu ainsi :

$$
\mathrm{P}=\frac{\mathrm{A} \times \mathrm{U}}{3180 \times 1^{\mathrm{r}}} \text { (Méthode Schmœger) }
$$

Formule dans laquelle :

$\mathrm{A}=$ déviation polarimétrique $\mathrm{D}$ traduite en minutes.

$3180=$ pouvoir rotatoire du lactose (53 ${ }^{\circ}$ d'après PARKINs et Tollens) exprimé en minutes.

$1^{\mathbf{r}}=$ longueur du tube polarimétrique exprimée en décimètres.

Le lactose $\mathrm{P}$ correspond à $\mathrm{U}$ centimètres cubes de filtrat, c'est-àdire à $25 \mathrm{~cm}^{3}$ de lait primitif : le taux $\mathrm{X}$ pour $100 \mathrm{~cm}^{3}$ de lait primitif s'obtient en multipliant $\mathrm{P}$ par 4.

Lait $\mathrm{n}^{\circ} 1$, individuel, en période avancée de lactation :

$$
\mathrm{D}=3^{\circ} 00^{\prime} \quad \mathrm{A}=180^{\prime} \quad \mathrm{P}=1,06 \quad \mathrm{X}=4,24
$$

Lait $\mathrm{n}^{\circ} 2$, collectif de mélange :

$$
\mathrm{D}=3^{\circ} 18^{\prime} \quad \mathrm{A}=198^{\prime} \quad \mathrm{P}=1,19 \quad \mathrm{X}=4,77
$$

Lait $\mathrm{n}^{\circ} 3$, collectif de mélange :
$\mathrm{D}=3^{022}$
$\mathrm{A}=202$
$\mathrm{P}=1,71$
$\mathrm{X}=4,86$

Les poids de lactose que nous avons déterminés par voie polarimétrique sont tout à fait comparables aux résultats trouvés par d'autres méthodes par les auteurs que nous avons consultés :

$4,60 \%$ d'après Maymone ; $3,94 \%$ d'après Diaconu ; $4,86 \%$ d'après Pappel et Hogan ; $5,00 \%$ d'après Meggit et Mann HA- 
RoLd ; 4,50\% d'après Marsella ; $5,17 \%$ d'après Martinoli; $4,80 \%$ d'après Batntiner et IrK ; $3,75 \%$ d'après Levine.

Un minimum de $2,33 \%$ a, en outre, été rencontré par BAINTER et IRK chez une bufflesse à terme de lactation.

Des données analytiques ci-dessus, il résulte que le contenu en lactose du lait de bufflesse varie relativement peu et comporte des valeurs assez voisines de celles du lactose contenu dans le lait de vache.

Cendres. - Nous avons obtenu les cendres par la méthode habituelle de l'incinération au rouge sombre de $10 \mathrm{~cm}^{3}$ de lait dans une capsule tarée.

Lait $\mathrm{n}^{0} 1: \mathrm{C}=0,99$ pour $100 \mathrm{~cm}^{3}$ (déduction faite du bichromate).

Lait $\mathrm{n}^{0} 2: \mathrm{C}=0,91$ pour $100 \mathrm{~cm}^{3}$.

Lait $n^{0} 3: \mathrm{C}=0,91$ pour $100 \mathrm{~cm}^{3}$ (déduction faite du bichromate) (Compte tenu de la volatilisation des chlorures.)

Le contenu en cendres des laits purs et normaux de bufflesse est, le plus souvent, selon les auteurs de 7,5 à 11 grammes par litre, tandis que le lait de vache n'en contient guère que 6,5 à 8 grammes.

D'après les recherches de TARTLER, la composition chimique des cendres du lait de bufflesse serait (moyenne de 3 analyses) : $\mathrm{K}^{2} \mathrm{O}=17,32 \% ; \mathrm{Na}^{2} \mathrm{O}=8,17 \% ; \mathrm{CaO}=33,51 \% ; \mathrm{MgO}=4,63 \%$; $\mathrm{Cl}=7,19 \% ; \mathrm{P}^{2} \mathrm{O}^{5}=33,50 \%$.

Tartuer dit que par suite du haut contenu du lait de bufflesse en $\mathrm{Ca}$ et en $\mathrm{P}$, ce lait est extrêmement utile pour la formation du squelette des enfants, et pour le traitement de la décalcification de l'organisme consécutive à certaines maladies.

Constante "lactose $+\mathbf{n}$ fois les cendres ". - D'après les données fournies par les analyses de plusieurs auteurs et les nôtres, nous avons calculé spécialement pour le lait de bufflesse la réplique de la constante chimique Bouin, qui relie, pour le lait de vache, le lactose aux cendres selon la formule $\mathrm{L}+5 \mathrm{C}=$ Constante.

Selon les résultats que nous avons trouvés, il faudrait adopter, pour le lait de bufflesse : $\mathrm{L}+6 \mathrm{C}=\mathrm{C}$-te 10,25 .

Les variations vont de 9,70 à $10,70 \mathrm{chez}$ la bufflesse, et nous pensons que des chiffres inférieurs à 9,70 ou supérieurs à 10,70 indiquent très nettement soit un mouillage, soit une addition de corps étranger, ce qui permettrait de reconnaître les fraudes par $\mathrm{L}+6 \mathrm{C}$. La moyenne 10,25 , que nous avons adoptée, a été déterminée avec des chiffres très hétérogènes concernant des races buffalines diverses élevées dans des climats divers. Il est bien entendu que pour chaque race considérée dans chaque région, il est nécessaire de déterminer très exactement la moyenne et les limites de variation de cette constante au cours de la lactation pour pouvoir affirmer la fraude. 
La constante Bouin, adaptée par nous au lait de bufflesse, est toujours préférable à la constante Bordas et Touplain :

$$
\text { Ed - Mat. prot. tot. }=\mathrm{L}+\mathrm{C}=\mathrm{C} \text {-te. }
$$

Chlorures. - On peut doser par les méthodes habituelles la teneur des cendres en acide phosphorique, chaux ou chlorures; mais, pour ces dernières, il y a eu des pertes par volatilisation. Or, le dosage exact des chlorures est indispensable quand on veut déterminer la constante moléculaire simplifiée (ef. paragraphe suivant). Nous avons, par conséquent, préféré opérer par voie humide, en modifiant légèrement l'excellente méthode Charpentier-Wollhart, préconisée pour le lait de vache par AUGUET, et qui consiste à préparer préalablement un lactosérum par précipitation du coagulum à l'aide du métaphosphate de soude, puis à doser dans ce sérum par le sulfocyanure de potassium N/10 la partie en excès (non fixée à l'état de chlorure d'argent et de nitrate de soude) du nitrate d'argent N/10 que l'on a adđditionné à la solution en quantité connue.

Afin d'obtenir une précipitation convenable du coagulum dans le lait de bufflesse, nous avons dû changer les proportions relatives de lait et de réactif usitées par Auguet pour le lait de vache.

Voici notre mode opératoire : $10 \mathrm{~cm}^{3}$ de lait de bufflesse sont introduits dans un ballon de $200 \mathrm{~cm}^{3}$. Nous ajoutons $6 \mathrm{~cm}^{3}$ de métaphosphate de soude à $5 \%$, et $1 / 2 \mathrm{~cm}^{3}$ d'acide acétique concentré ; nous complétons avec de l'eau distillée à $200 \mathrm{~cm}^{3}$ (et ajoutons, si nécessaire, au surplus $1 \mathrm{~cm}^{3}$ de sous-acétate de plomb pour précipiter le bichromate dont la coloration jaune gêne le virage final). Nous agitons et filtrons. Ensuite, nous prélevons $100 \mathrm{~cm}^{3}$ de cette liqueur correspondant à $5 \mathrm{~cm}^{3}$ de lait (le volume du sous-acétate introduit est négligeable, car il représente très sensiblement celui de l'insoluble de la prise d'essai) et nous y ajoutons $3 \mathrm{~cm}^{3}$ d'acide azotique concentré et $5 \mathrm{~cm}^{3}$ de nitrate d'argent. Le mélange est divisé en deux parties égales de $54 \mathrm{~cm}^{3}$ chacune que nous filtrons et analysons à part pour une plus grande exactitude, quitte à prendre la moyenne des résultats obtenus.

Chacun des deux filtrats, qui correspond à $2 \mathrm{~cm}^{3} 5$ de lait primitif et à $2 \mathrm{~cm}^{3} 50$ de nitrate d'argent employé, est reçu dans une fiole conique de $125 \mathrm{~cm}^{3}$, nous mettons le réactif coloré (trois gouttes d'alun de fer ammonical). Nous titrons l'excès de liqueur argentique par le sulfocyanure de potassium N/10. La fin de la réaction est indiquée par une teinte jaune persistante (formation de sulfocyanure de fer). La teneur en $\mathrm{NaCl}$ de $2 \mathrm{~cm}^{3} 5$ de lait est égale à $(2,5-n) \times$ 0,005846 ; ce dernier chiffre représente le poids de $\mathrm{NaCl}$ correspondant à $1 \mathrm{~cm}^{3}$ de $\mathrm{NO}^{3} \mathrm{Ag}(\mathrm{N} / 10)$, et $2,5-n$ c'est la différence entre le nitrate d'argent employé et l'excès $n$ restant dans la solution, qui est titré par le volume de sulfocyanure de potassium écoulé. 
Nous faisons exactement le même dosage pour le contenu de la seconde fiole et, en multipliant par 40 la moyenne des deux résultats trouvés pour $2 \mathrm{~cm}^{3} 5$ de lait, nous obtenons le taux de $\mathrm{NaCl}$ pour $100 \mathrm{~cm}^{3}$ de lait de bufflesse.

Les chiffres trouvés par nous ont été respectivement :

Lait $\mathrm{n}^{0} 1$ (individuel) : 0,114 gr. pour $100 \mathrm{~cm}^{3}$.

Lait no 2 (de mélange) : 0,104 gr. pour $100 \mathrm{~cm}^{3}$.

Lait $\mathrm{n}^{0} 3$ (de mélange) : 0,103 gr. pour $100 \mathrm{~cm}^{3}$.

La teneur élevée du lait de bufflesse en chlorures, ainsi qu'il résulte de nos analyses et de celles de TARTLER, expliquerait la nécessité, pour cet animal, d'absorber une plus forte dose de sel avec ses aliments que celle consommée par la vache.

Constante moléculaire simplifiée réelle. - MATHIEU et FeRRÉ ont établi pour le lait de vache, en 1914, une constante moléculaire simplifiée qui est certainement l'une des meilleures constantes physico-chimiques que nous connaissions pour déceler les laits fraudés. Cette constante repose sur le balancement osmotique mis en évidence par Ch. Porcher (1906) - qui existe entre le lactose et le chlorure de sodium (on néglige les citrates, phosphates, sulfates, bicarbonates, l'azote non prótéique et l'ensemble salin des colloïdes); elle est représentée par la somme du lactose hydraté et de l'équivalent isotonique en lactose des chlorures exprimés en $\mathrm{NaCl}$ : 1 gramme de sel marin équivaut à 11,9 de lactose. La C. M. S. brute trouvée doit être ramenée au volume du lait, diminué de celui des insolubles (caséine et matière grasse), pour avoir :

(C. M. S. réelle $\Rightarrow$ L p. $100 \mathrm{~cm}^{3}+\mathrm{NaCl}$ p. $100 \mathrm{~cm}^{3} \times 11,9 \frac{100}{100-\mathrm{V}}$

$\mathrm{V}$ est le volume des insolubles calculé comme précédemment : ef. paragraphe Lactose. Dans les trois laits de bufflesse sur lesquels ont porté nos expériences, la valeur de la C. M. S. R. a été la suivante :

$$
\begin{aligned}
& \text { Lait } \mathrm{n}^{\circ} 1 \text { C. M. S. R. }=(4,24+1,36) 100: 82,20=6,81 \% \mathrm{~cm}^{3} . \\
& \text { Lait } \mathrm{n}^{\circ} 2 \text { C. M. S. R. }=(4,77+1,24) 100: 83,42=7,20 \% \mathrm{~cm}^{3} . \\
& \text { Lait } \mathrm{n}^{\circ} 3 \text { C. M. S. R. }=(4,86+1,22) 100: 83,42=7,28 \% \mathrm{~cm}^{3} .
\end{aligned}
$$

Les chiffres que nous avons obtenus pour le lait de bufflesse restent, somme toute, dans la limite de la C. M. S. R. du lait de vache $(6,5$ à 7,5 , soit 65 à 75 par litre). - Dans les laits fraudés par addition d'eau, cette constante subit un abaissement proportionnel permettant à l'expert de déceler la tromperie.

Acide citrique. - "L'acide citrique, comme on sait, est un constituant normal du lait. Il a été rencontré par divers chimistes 
dans le lait de vache, dans la quantité de 0,1 à $0,2 \%$, et il se trouve, selon FleischmanN, à peu près dans la même proportion dans le lait de bufflesse. "

Dans deux analyses qu'a faites le Professeur MAymone en adoptant la méthode proposée par DenIĠ̀s pour la recherche de l'acide citrique dans le lait de vache, ce savant a trouvé respectivement $0,22 \%$ d'acide citrique pour un échantillon de lait de bufflesse ayant une densité de 1032,4 et un contenu en graisse de 8,62 pour $100 \mathrm{~cm}^{3}$; et, d'autre part, $0,27 \%$ d'acide citrique pour un autre lait ayant une densité de 1033,2 et un taux de matière grasse de 7,43 pour $100 \mathrm{~cm}^{3}$.

Note. - Certaines considérations qui précèdent résultent des expériences officielles de contrôle laitier et beurrier buffalin, auxquelles nous avons nousmême participé, ou que nous avons simplement suivies durant les années 1933-1934-1935, en Italie (provinces de Rome, Naples et Salerne). Nous avons, d'autre part, apporté une importante contribution personnelle aux recherches antérieures par l'étude approfondie d'un certain nombre d'échantillons de laits de bufflesse individuels et de mélange, que M. le Professeur Bartolo. MAYMONE, directeur de I'Istituto Sperimentale Zootecnico de Rome, nous a fait parvenir, aux fins d'analyse, au Laboratoire de l'Ecole Supérieure de Laiterie de l'Université de Nancy (conservation des échantillons par le bichromate de potassium à raison de $1 \mathrm{~cm}^{3}$ de solution à $8 \%$ par litre, ou par le formol à raison de $1 \mathrm{~cm}^{3}$ de solution à $40 \%$ par litre). Les analyses ont été effectuées sous le contrôle effectif de M. le Professeur Bouin et de son assistante $\mathrm{M}^{\text {me }} \mathrm{D}^{\mathrm{r}}$ Waissmann-Karlin.

Nous avons dû modifier quelques-unes des méthodes normalement employées pour le lait de vache afin de pouvoir les appliquer au lait de bufflesse (recherche du lactose par la méthode Bouin, des chlorures par la méthode Charpentier-Wollhart). Enfin, nous nous sommes appliqué à calculer, pour ce lait, des constantes physico-chimiques correspondant à celles de Gros, Cornatba, Bordas et Touplain, Mathieu et Ferré, Bouin, et des formules empiriques analogues à celles précédemment imaginées pour déterminer commodément la proportion approximative de certains composants chimiques des autres laits.

\section{CONCLUSIONS GÉNÉRALES}

Nous reportons dans le tableau ci-dessous les données relatives aux compositions chimiques des laits de vache, de chèvre et de brebis (d'après A. Monvoisin) comparées à celle du lait de bufflesse (limites extrêmes résultant des travaux de Maymone, Pizzi, Stazi, Rimini, Martinoli, Marsella, Gidliani en Italie; de Fleischmann, Tartler et H. Rievel en Allemagne; de Rosenberger, Baintner et Irk, Sierban et Koerfer, Gorup-Besanez en Hongrie ; de Diaconu et Iliescu en Roumanie; de Pappel et Hogan en Egypte; de Megrit et ManN Harod aux Indes; de Levine en Chine (Canton) et de nous-même) : 


\begin{tabular}{|c|c|c|c|c|}
\hline$\%$ & Vache & Chèvre & Brebis & Bufflesse \\
\hline Matière grasse & $1,69-7,60$ & $2,45-5,73$ & $3,70-10,40$ & $5,00-17,00$ \\
\hline Substances azotées .. & $4,30-8,50$ & $2,25 \cdot 7,07$ & $5,10-9,00$ & $3,16-9,50$ \\
\hline Lactose ............ & $2,84-7,66$ & $2,59-5,46$ & $4,10-5,80$ & $2,33-5,28$ \\
\hline Cendres $\ldots \ldots \ldots \ldots$ & $0,49-1,16$ & $0,54-1,11$ & $0,55-1,00$ & $0,70-1,30$ \\
\hline Eau...., & $75,27-91,17$ & $82,69-90,91$ & $77,00-87,30$ & $72,95-84,19$ \\
\hline
\end{tabular}

En outre, nous reproduisons le tableau très caractéristique dressé par G. M. ItIescu pour la composition chimique comparée des laits de vache et de bufflesse en Roumanie :

\begin{tabular}{|c|c|c|c|c|c|c|}
\hline \multirow{2}{*}{$\begin{array}{c}\% \\
\text { (Poids spécif.) }\end{array}$} & \multicolumn{3}{|c|}{ Vache } & \multicolumn{3}{|c|}{ Bufflesse } \\
\hline & $\begin{array}{l}\text { min. } \\
(1.030)\end{array}$ & moy. & $\begin{array}{l}\max . \\
(1.034)\end{array}$ & $\min$. & $\begin{array}{l}\text { moy. } \\
\text { (1.031) }\end{array}$ & $\begin{array}{l}\max . \\
(1.035)\end{array}$ \\
\hline ère grasse $\ldots$. & 3,10 & 5,50 & 8,10 & 5,00 & 7,80 & 15,00 \\
\hline tances azotées. & 4,80 & 6,65 & 8,50 & 6,37 & 7,93 & 9,50 \\
\hline sse ......... & 3,48 & 3,96 & 4,34 & 3,30 & 3,94 & 4,59 \\
\hline $\operatorname{ces} \ldots .$. & 0,70 & - & 1,30 & 0,80 & 1,05 & 1,30 \\
\hline$\ldots \ldots \ldots \ldots$ & 83,55 & 85,50 & 91,50 & 76,10 & 79,55 & 83,00 \\
\hline
\end{tabular}

Il résulte des chiffres comparatifs ci-dessus, .que tous les composants normaux du lait de vache se retrouvent, évidemment, dans le lait de bufflesse, mais les proportions relatives dans lesquelles ces eomposants existent dans le lait de bufflesse sont quelque peu différentes, et tout à l'avantage de ce dernier.

On est immédiatement frappé, si l'on consulte l'un ou l'autre des deux tableaux précédents, par la teneur plus faible en eau du lait de bufflesse, c'est-à-dire par sa plus forte teneur en extrait sec, qui est due, comme on le voit, à la plus grande richesse de ce lait en matière grasse et en substances azotées.

Il est donc certain que le lait de bufflesse est beaucoup plus nutritif que le lait de vache, à volume égal. Cependant, comme il est encore plus gras que ce dernier, il est nécessaire de le materniser par addition d'une bonne moitié d'eau bouillie sucrée, si l'on veut l'administrer aux nourrissons : parfois même la matière grasse est si abondante qu'un tel coupage est insuffisant et une dilution plus grande appaurrirait le mélange en ce qui concerne les autres composants chimiques. Le lait de bufflesse est excellent pour l'enfant sevré.

En somme, le lait de bufflesse a plus ou moins les propriétés du lait de brebis, sans en avoir l'odeur épouvantable. Nous savons en outre que la bufflesse est rarement tuberculeuse et qu'elle est réfractaire à plusieurs maladies transmissibles à l'homme : son lait est le plus salubre qui soit, et sa consommation régulière à l'état naturel, de fromage ou de yogourt, passe pour donner une longévité exceptionnelle aux habitants des pays balkaniques et de la péninsule turque. 
Malheureusement, rien n'est plus facile que de falsifier du lait de bufflesse avec du lait de vache, et, bien entendu, les éleveurs et les laitiers ne manquent pas de le faire : "Le lait de vache, dit MAYMone, peut être adjoint à un lait de bufflesse assez riche en graisse, en quantité voulue pour abaisser le taux de cette matière jusqu'à la teneur minimum assignée par la loi au lait de bufflesse $(6 \%)$ sans que l'analyse - en se basant sur les propriétés physiques et chimiques puisse sûrement révéler la fraude.

"Papper et Hogan ont recouru à la méthode biologique des précipitations spécifiques, obtenant des résultats assez satisfaisants. Mais cette méthode exige une technique de laboratoire qui ne se prête guère aux applications dans la pratique industrielle. ")

(A suivre).

\title{
A PROPOS DE LA THÉORIE DE LA DÉSACIDIFICATION ÉLECTRIQUE DU LAIT
}

\author{
par \\ le Professeur Dr Otтo GRATK
}

La revue "Le Lait", dans son numéro 151 de janvier 1936, a publié, sous le titre "A propos de la désacidification électrique du lait ", une étude de MM. Jean Pien, directeur, et Jacques BAIsse, ingénieur chimiste, des laboratoires des Fermiers Réunis.

Dans cette étude, les auteurs donnent une nouvelle explication des phénomènes, des réactions qui se produisent pendant la désacidification électrique du lait.

Ils contestent que la désacidification soit due à une décomposition de l'acide lactique par électrolyse et affirment qu'elle résulte de ce fait que les bases alcalino-terreuses sont mises en liberté - aux dépens surtout des phosphates lactiques et même des caséinates de phosphore - et viennent neutraliser l'acide lactique et une partie de la caséine.

C'est à ces mêmes bases libérées qu'il faudrait attribuer le fait que l'on peut rendre basique un lait traité par électrolyse et qui ne contient done plus d'acide lactique.

D'après la conception de MM. PIEn et BAISSE, la désacidification électrique du lait reposerait surtout sur la transformation électrolytique en phosphates d'aluminium des phosphates existants.

A cette théorie - que les auteurs eux-mêmes présentent d'ailleurs à titre hypothétique - on peut opposer ce qui suit :

Des essais, dont les résultats n'ont pas été publiés jusqu'à présent, mais ont été confirmés par des milieux compétents, ont prouvé que la désacidification électrique du lait pouvait parfaitement être 